\title{
Hydrogen Production by Catalytic Partial Oxidation of Coke Oven Gas in $\mathrm{BaCo}_{0.7} \mathrm{Fe}_{0.3-x} \mathrm{Zr}_{x} \mathrm{O}_{3-\delta}$ Ceramic Membrane Reactors
}

\author{
Weilin $\mathrm{YAO}^{1}$, Hongwei $\mathrm{CHENG}^{1,2, \mathrm{a}^{*}}$, Pengfei $\mathrm{WANG}^{1}$, Xionggang $\mathrm{LU}^{1,2, \mathrm{~b}}$, Xingli ZOU ${ }^{1,2}$ \\ and Qian XU ${ }^{1,2}$ \\ ${ }^{1}$ School of Materials Science and Engineering, Shanghai University, Shanghai 200072, China \\ ${ }^{2}$ State Key Laboratory of Advanced Special Steel, Shanghai University, Shanghai 200072, China \\ ahwcheng@shu.edu.cn, bluxg@shu.edu.cn
}

\begin{abstract}
The $\mathrm{BaCo}_{0.7} \mathrm{Fe}_{0.3-x} \mathrm{Zr}_{x} \mathrm{O}_{3-\delta}$ (BCFZ, $\left.x=0.04-0.12\right)$ mixed ionic-electronic conducting (MIEC) membranes were synthesized with a sol-gel method and evaluated as potential membrane reactor materials for the partial oxidation of coke oven gas (COG). The effect of zirconium content on the phase structure, microstructure and performance of the BCFZ membrane under $\mathrm{He}$ or $\mathrm{COG}$ atmosphere were systemically investigated. The $\mathrm{BaCo}_{0.7} \mathrm{Fe}_{0.24} \mathrm{Zr}_{0.06} \mathrm{O}_{3-\delta}$ membrane exhibited the best oxygen permeability and good operation stability, which could be a potential candidate of the membrane materials for hydrogen production through the partial oxidation of COG.
\end{abstract}

\section{Introduction}

Hydrogen is regarded as the most effective solution to the increasingly serious problems of environmental pollution and depleting energy sources in the 21 st century. Currently, hydrogen is mainly produced from fossil fuels and chemical by-products $[1,2]$. Of these methods, coke oven gas (COG), a by-product generated during the production of coke from coal, has attracted much interest as one of the most promising high quality sources of hydrogen $[3,4]$. The separation of oxygen from air and catalytic partial oxidation of methane $(\mathrm{POM})$ in $\mathrm{COG}$ has been achieved in a mixed ionic-electronic conducting (MIEC) membrane reactor, which significantly reduces the energy consumption and cost of hydrogen production compared to the conventional processes [5].The COG exhibits strong reducibility because its main components are $54-59 \% \mathrm{H}_{2}, 24-31 \% \mathrm{CH}_{4}$, and 5.5-7\% $\mathrm{CO}$ [6]. Therefore, structural stability under a reductive atmosphere and high oxygen permeability are expected for a MIEC membrane to meet the requirements of practical applications. The

* Corresponding author:hwcheng@shu.edu.cn 
perovskite-type materials of $\mathrm{ABO} 3$ composition have attracted much interest due to their excellent oxygen permeability.

In this work, a series of $\mathrm{BaCo}_{0.7} \mathrm{Fe}_{0.3-x} \mathrm{Zr}_{x} \mathrm{O}_{3-\delta}(\mathrm{BCFZ}, x=0.04-0.12)$ MIEC membranes were synthesized with a sol-gel method. Their phase structure, microstructure and performance under He or COG atmosphere were systematically investigated.

\section{Materials and Methods}

The $\mathrm{BaCo}_{0.7} \mathrm{Fe}_{0.3-x} \mathrm{Zr}_{x} \mathrm{O}_{3-\delta}(x=0.04-0.12)$ powders were synthesized with a sol-gel method as described in our previous work [7]. The primary powders were calcined at $900{ }^{\circ} \mathrm{C}$ for $5 \mathrm{~h}$ to obtain the powder with the aforementioned final composition. The green disks were prepared under a hydraulic pressure of $150-200 \mathrm{MPa}$ and then sintered at $1110{ }^{\circ} \mathrm{C}$ for $8 \mathrm{~h}$ in ambient air.

The $\mathrm{Ni} / \mathrm{MgAl}_{2} \mathrm{O}_{4}$ catalyst was prepared with urea-template and impregnation method. Stoichiometric amounts of $\mathrm{Mg}\left(\mathrm{NO}_{3}\right)_{2} \cdot 6 \mathrm{H}_{2} \mathrm{O}$ and $\mathrm{Al}\left(\mathrm{NO}_{3}\right)_{3} \cdot 9 \mathrm{H}_{2} \mathrm{O}$ were dissolved in deionized water, followed by the introduction of a defined amount of urea. The mole ratio of urea:total nitrate was 3:1. A certain amount of the triblock copolymer P123, which was to act as a template, was dissolved in deionized water in another vessel with vigorous stirring. The P123: $\mathrm{MgAl}_{2} \mathrm{O}_{4}$ mass ratio was about 3:2. After the urea and $\mathrm{P} 123$ were completely dissolved, the solutions in the two vessels were mixed together. The obtained mixture was then stirred vigorously for $24 \mathrm{~h}$, dried at $110{ }^{\circ} \mathrm{C}$ overnight, and then calcined at $850{ }^{\circ} \mathrm{C}$ for $5 \mathrm{~h}$ in air to form a white powder composed of $\mathrm{MgAl}_{2} \mathrm{O}_{4}$. The $\mathrm{MgAl}_{2} \mathrm{O}_{4}$ support was impregnated into a nickel nitrate solution made from $\mathrm{Ni}\left(\mathrm{NO}_{3}\right)_{2} \cdot 6 \mathrm{H}_{2} \mathrm{O}$ and left overnight at room temperature, followed by drying at $110^{\circ} \mathrm{C}$ and calcination at $850{ }^{\circ} \mathrm{C}$ for $2 \mathrm{~h}$ in air. The Ni mass fraction in the $\mathrm{Ni} / \mathrm{MgAl}_{2} \mathrm{O}_{4}$ catalyst was $10 \mathrm{wt} \%$. The $\mathrm{Ni} / \mathrm{MgAl}_{2} \mathrm{O}_{4}$ catalyst powder was pressed into a bulk material in a stainless steel mold under a hydraulic pressure of $40 \mathrm{MPa}$. The bulk catalyst was subsequently broken down into 20-40 mesh granules for later use.

The phase structure of the synthesized BCFZ membranes was examined in a Bruker-AXS D8 ADVANCE diffractometer, with $\mathrm{Cu}$ radiation $(40 \mathrm{kV}, 40 \mathrm{~mA})$. The cross-section morphology of the fresh BCFZ membranes was studied with a field emission scanning electron microscope (JEOL JSM-6700F) at an excitation voltage of $15 \mathrm{kV}$. The performance of the disk-shaped membranes under $\mathrm{He}$ or COG atmosphere was measured using a home-made equipment as described in our previous work [8].

\section{Results and Discussion}

The XRD patterns of the as-synthesized $\mathrm{BaCo}_{0.7} \mathrm{Fe}_{0.3-x} \mathrm{Zr}_{x} \mathrm{O}_{3-\delta}(x=0.04-0.12)$ membranes are shown in Fig. 1(a). All of the BCFZ membranes exhibit a pure cubic perovskite structure, except for $x=0.12$ with traces of a $\mathrm{BaZrO}_{3}$ phase. Fig. 1(e)-(f) present the microstructure of the fresh BCFZ membranes. All the sintered membranes show a dense ceramic structure with a small amount of enclosed pores. Therefore, these synthesized BCFZ membrane can be used for the oxygen separation. 

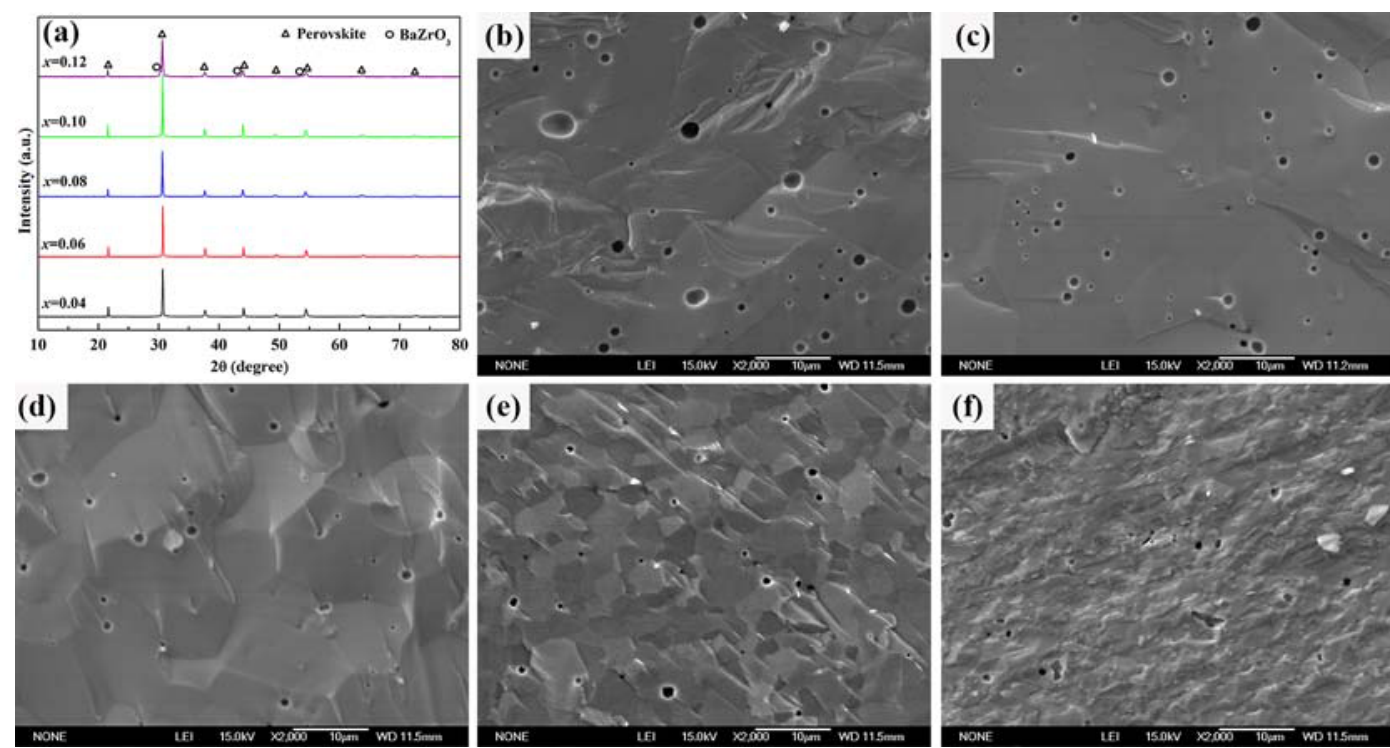

Fig. 1 - (a) The XRD patterns of the fresh BCFZ membranes; the cross section SEM images of the fresh BaCo0.7Fe0.3-xZrxO3- $\delta$ membranes: (b) $\mathrm{x}=0.04$, (c) $\mathrm{x}=0.06$, (d) $\mathrm{x}=0.08$, (e) $x=0.10$, (f) $x=0.12$.

The time dependence of oxygen permeation flux through the BCFZ membranes under pure He atmosphere at different temperature is shown in Fig. 2(a). The oxygen permeation fluxes of all the samples increase with temperatures because of the increased diffusion, surface exchange reaction rate [9]. At the low temperature $\left(<800^{\circ} \mathrm{C}\right)$, the oxygen permeation fluxes of all the membranes drops gradually with the prolongation of time, which may be caused by the phase transformation [10]. Of all the samples, the highest average oxygen permeation flux of $2.72 \mathrm{ml} \cdot \mathrm{min}^{-1} \cdot \mathrm{cm}^{-2}$ at $925^{\circ} \mathrm{C}$ under pure He atmosphere was achieved by the $x=0.06 \mathrm{BCFZ}$ membrane.

In order to study the potential application of BCFZ membranes in the production of hydrogen from COG, the performance of the membranes under a COG atmosphere was investigated. The catalytic partial oxidation of methane in COG was conducted in the BCFZ membrane reactor packed with a $\mathrm{Ni} / \mathrm{MgAl}_{2} \mathrm{O}_{4}$ catalyst on the permeation side.
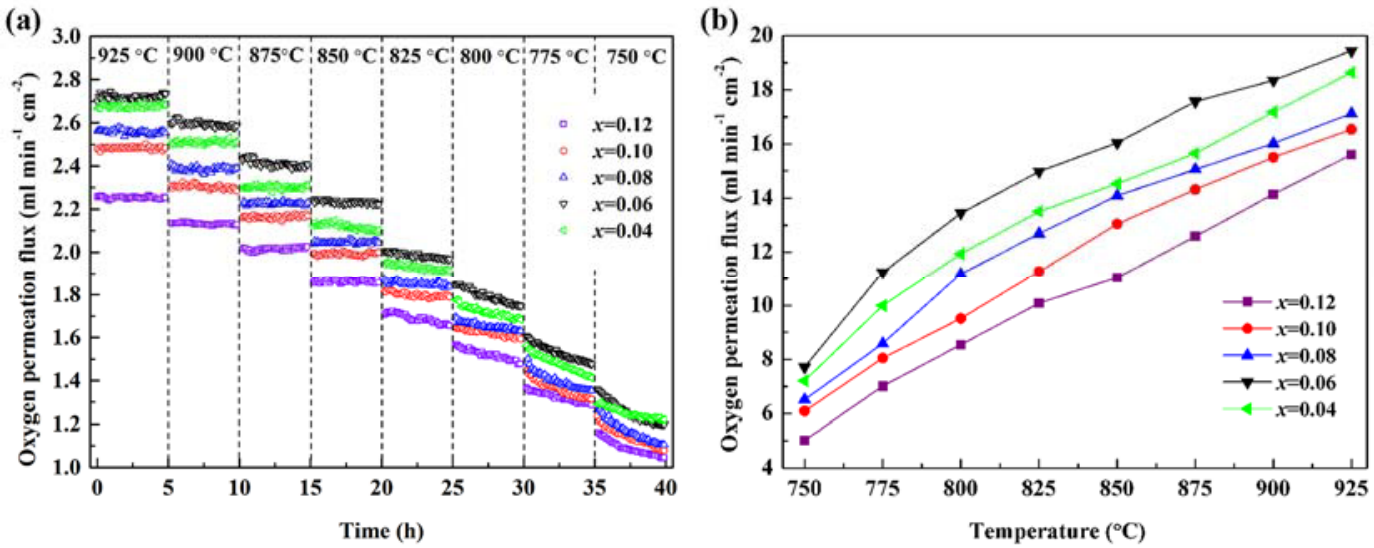

Fig.2 - (a) The time dependence of the oxygen permeation flux through BCFZ membranes under an air/pure He gradient and (b) The temperature dependence of the oxygen permeation flux through BCFZ membranes an air/COG gradient: membrane thickness $=1.0 \mathrm{~mm}$; air $=300 \mathrm{ml} \min -1$; pure $\mathrm{He}=300 \mathrm{ml}$ min-1; COG $=100 \mathrm{ml} \cdot \mathrm{min}-1$. 
(a)

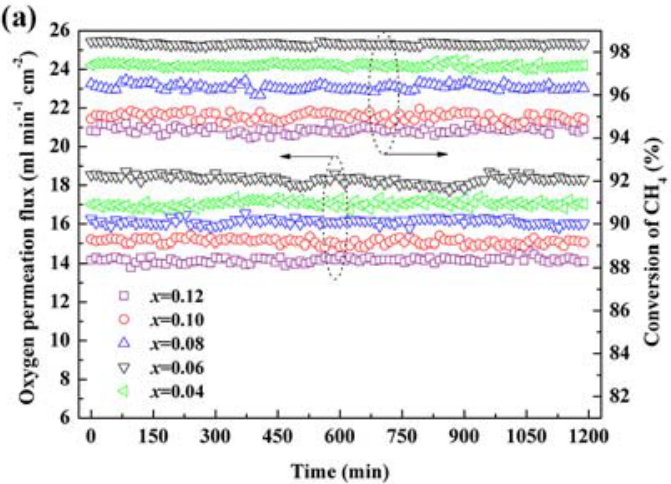

(c)

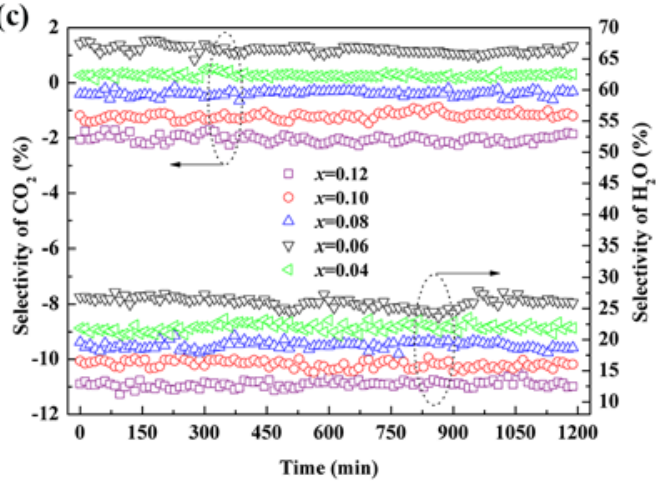

(b)

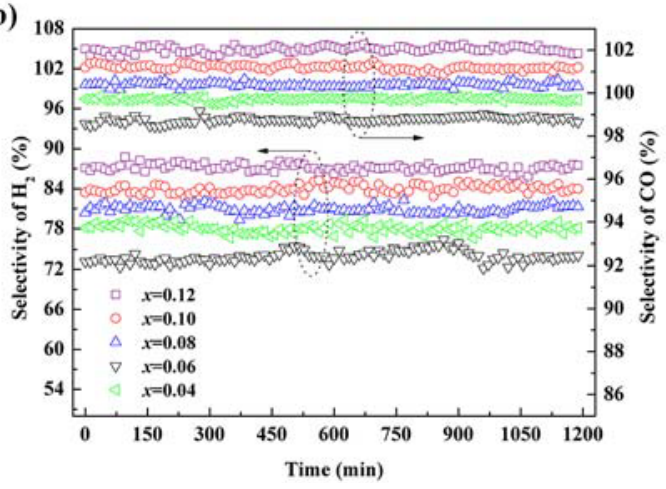

(d)

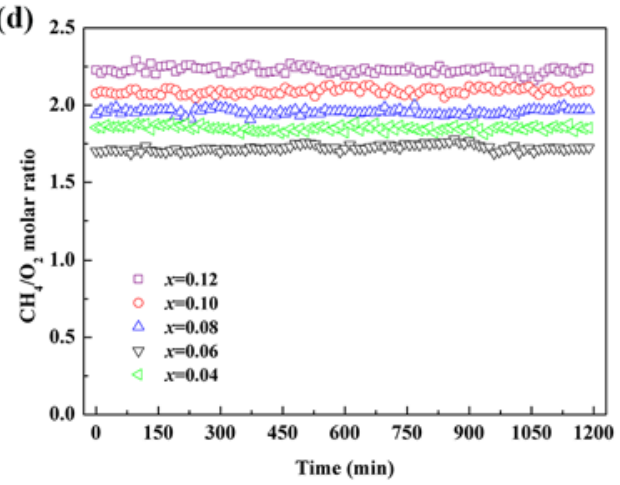

Fig. 3 - The performance of BCFZ membrane reactors for the catalytic partial oxidation of COG: (a) the oxygen permeation flux and $\mathrm{CH} 4$ conversion; (b) the selectivity of $\mathrm{H} 2$ and $\mathrm{CO}$; (c) the selectivity of $\mathrm{H} 2 \mathrm{O}$ and $\mathrm{CO}$; (d) the $\mathrm{CH} 4 / \mathrm{O} 2$ molar ratio. Reaction conditions: $\mathrm{COG}=100 \mathrm{ml}$ min- 1 ; air $=300 \mathrm{ml} \mathrm{min}-1$; membrane thickness $=1.0 \mathrm{~mm}$; temperature $=900^{\circ} \mathrm{C}$.

Fig. 2(b) shows the temperature dependence of the oxygen permeation flux through the 1-mm-thick BCFZ $(x=0.04-0.12)$ membranes under an air/COG gradient. The oxygen permeation fluxes of all the membranes were considerably high, i.e., several times or even one order of magnitude higher than that in the air/He gradient. This enhancement might be caused by the generation of more oxygen vacancies under the lower oxygen partial pressure on the permeation side because of the rapid reaction between the COG and penetrating oxygen. In addition, the oxygen permeation flux of the BCFZ membranes first increases, and then decreases with increasing $\mathrm{Zr}$ content, which is consistent with the oxygen flux behavior under an air/He gradient. Similar to the results under an air/He gradient, the highest oxygen permeation flux of $19.4 \mathrm{ml} \cdot \mathrm{min}^{-1} \cdot \mathrm{cm}^{-2}$ at $925{ }^{\circ} \mathrm{C}$ was achieved by the $x=0.06 \mathrm{BCFZ}$ membrane. Hence, it can be concluded that the oxygen permeability in the catalytic partial oxidation of COG in the BCFZ membrane reactor can be improved by the introduction of a certain amount of $\mathrm{Zr}$ to BCFZ membranes. 


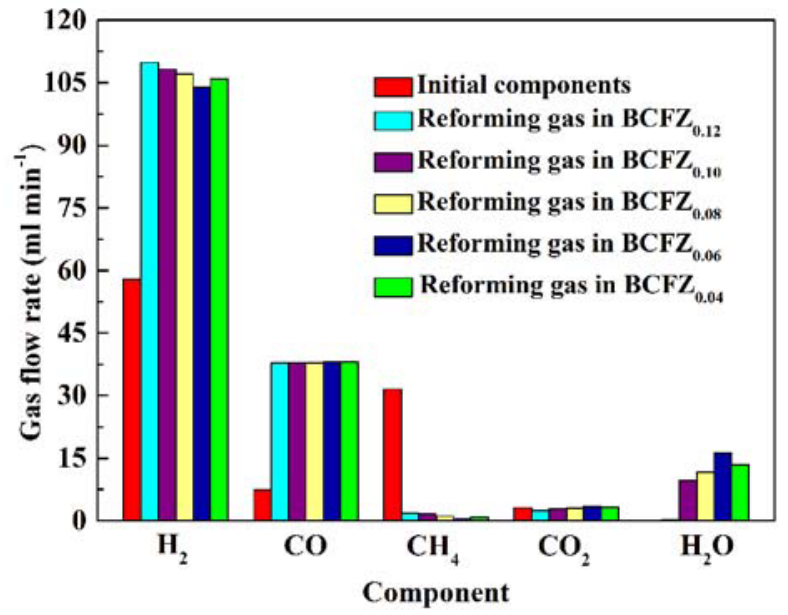

Fig. 4 Variation of components in the COG before and after undergoing catalytic reforming in BCFZ membrane reactors.

The effects of $\mathrm{Zr}$ content on the performance of the BCFZ membrane reactors at $900{ }^{\circ} \mathrm{C}$ are shown in Fig. 3. It can be seen that the oxygen permeation flux, $\mathrm{CH}_{4}$ conversion, and the product yields remain constant during $20 \mathrm{~h}$ tests of the BCFZ membrane reactors. In Fig. 3(a), the average oxygen permeation flux and $\mathrm{CH}_{4}$ conversion first increase and subsequently decrease with increasing $\mathrm{Zr}$ concentration. Of all the membranes, the $x=0.06 \mathrm{BCFZ}$ membrane reactor shows the highest average oxygen permeation flux of $18.3 \mathrm{ml} \cdot \mathrm{min}^{-1} \cdot \mathrm{cm}^{-2}$ and $\mathrm{CH}_{4}$ conversion of $98.4 \%$, while those of the $x=0.12$ membrane are the lowest at 14.2 $\mathrm{ml} \cdot \mathrm{min}^{-1} \cdot \mathrm{cm}^{-2}$ and $94.4 \%$, respectively. Furthermore, the product yields are affected by the different oxygen permeation fluxes and reactant conversions resulting from the different $\mathrm{Zr}$ contents of the BCFZ membranes. Fig. 3(b) shows the influence of $\mathrm{Zr}$ content on the selectivity of $\mathrm{H}_{2}$ and $\mathrm{CO}$. The average selectivity of $\mathrm{H}_{2}$ and $\mathrm{CO}$ changed from $73.9 \%$ and $98.8 \%(x=0.06)$ to $87.1 \%$ and $102.0 \%(x=0.12)$, respectively, which is in contrast to the oxygen permeation flux results. The decreased selectivity of $\mathrm{H}_{2}$ is attributed to the enhancement of reaction between $\mathrm{H}_{2}$ and permeated oxygen, which improves the $\mathrm{H}_{2} \mathrm{O}$ yields, as shown in Fig. 3(c) [11]. For the $x=0.08,0.10$, and 0.12 membranes, the CO selectivity exceeds $100 \%$ because of the partial conversion of $\mathrm{CO}_{2}$ in the $\mathrm{COG}$ to $\mathrm{CO}$ through the dry reforming reaction, which corresponds with the negative values of $\mathrm{CO}_{2}$ selectivity in Fig. 3(c) [12]. This phenomenon might be caused by the $\mathrm{CH}_{4} / \mathrm{O}_{2}$ molar ratio, as presented in Fig. 3(d). It can be seen that the average $\mathrm{CH}_{4} / \mathrm{O}_{2}$ molar ratio varies from 1.7 to 2.2 , indicating that the oxygen permeation flux varies from an excess of oxygen to a shortage of oxygen for the partial oxidation reaction. Moreover, after the catalytic reforming in the BCFZ membrane reactor (Fig. 4), the flow rates of $\mathrm{H}_{2}$ and $\mathrm{CO}$ were greatly improved, reaching 103.8-109.8 and 37.8-38.08 $\mathrm{ml} \cdot \mathrm{min}^{-1}$, respectively. As shown in Fig. 4, the amount of hydrogen after reforming is $1.8-1.9$ times the original hydrogen in the COG.

\section{Conclusions}

Mixed ionic-electronic conducting (MIEC) materials composed of $\mathrm{BaCo}_{0.7} \mathrm{Fe}_{0.3-x} \mathrm{Zr}_{x} \mathrm{O}_{3-\delta}$ (BCFZ, $x=0.04-0.12$ ) were synthesized with a sol-gel method. After being sintered at $1110{ }^{\circ} \mathrm{C}$ for $8 \mathrm{~h}$, a pure cubic perovskite structure was obtained for $x=0.04-0.10$. While, traces of a $\mathrm{BaZrO}_{3}$ phase were detected for $x=0.12$. All the BCFZ membranes exhibit a dense ceramic structure. The oxygen permeability of the BCFZ membranes increased with 
increasing operating temperature. The oxygen permeation flux first increased and then decreased with increasing $\mathrm{Zr}$ content under $\mathrm{He}$ and $\mathrm{COG}$ atmospheres. The $\mathrm{BaCo}_{0.7} \mathrm{Fe}_{0.24} \mathrm{Zr}_{0.06} \mathrm{O}_{3-\delta}$ membrane showed the highest oxygen permeation flux of 2.72 $\mathrm{ml} \cdot \mathrm{min}^{-1} \cdot \mathrm{cm}^{-2}$ at $925^{\circ} \mathrm{C}$ under an air/He gradient. The good long-term operation stability and highest permeation flux of $18.3 \mathrm{ml} \cdot \mathrm{min}^{-1} \cdot \mathrm{cm}^{-2}$ at $900{ }^{\circ} \mathrm{C}$ under an air/COG gradient were achieved in the $\mathrm{BaCo}_{0.7} \mathrm{Fe}_{0.24} \mathrm{Zr}_{0.06} \mathrm{O}_{3-\delta}$ membrane reactor, which is a promising membrane material for the catalytic partial oxidation of $\mathrm{COG}$ to produce hydrogen.

\section{Acknowledgments}

This work was supported by the National Natural Science Foundation of China (No. 51474145), the National Science Fund for Distinguished Young Scholars (No. 51225401), the Shanghai Rising-Star Program (15QA1402100) and Innovation Program of Shanghai Municipal Education Commission (No. 14YZ013).

\section{References}

1. T.J. Huang, M.C. Huang, M.S. Huang, Novel methane steam-reforming catalyst of $\mathrm{Ni}-\mathrm{Bi}_{2} \mathrm{O}_{3} / \mathrm{GDC}$ to reduce $\mathrm{CO}$ for hydrogen production, Applied Catalysis A: General. 354 (2009) 127-131.

2. J.R. Rostrup-Nielsen, Fuels and Energy for the Future: The Role of Catalysis, Catalysis Reviews. 46 (2004) 247-270.

3. F. Joseck, M. Wang, Y. Wu, Potential energy and greenhouse gas emission effects of hydrogen production from coke oven gas in U.S. steel mills, Int. J. Hydrogen Energy. 33 (2008) 1445-1454.

4. M.L. Murray, E. H. Seymour, J. Rogut, S. W.Zechowska, Stakeholder perceptions towards the transition to a hydrogen economy in Poland, Int. J. Hydrogen Energy. 33 (2008) 20-27.

5. Z.B. Yang, W.Z. Ding, Y.Y. Zhang, X.G. Lu, Y.W. Zhang, P.J. Shen, Catalytic partial oxidation of coke oven gas to syngas in an oxygen permeation membrane reactor combined with $\mathrm{NiO} / \mathrm{MgO}$ catalyst, Int. J. Hydrogen Energy. 35 (2010) 6239-6247.

6. P.J. Kirton, J. Ellis, P.T. Crisp, The analysis of organic matter in coke oven emissions, Fuel. 70 (1991) 1383-1389.

7. B. Jiang, H.W. Cheng, L.F. Luo, X.G. Lu, Z.F. Zhou, Oxygen Permeation and Stability of $\mathrm{Ce}_{0.8} \mathrm{Gd}_{0.2} \mathrm{O}_{2-\delta}-\mathrm{PrBaCo}_{2-x} \mathrm{Fe}_{x} \mathrm{O}_{5+\delta}$ Dual-phase Composite Membranes, Journal of Materials Science \& Technology. 30 (2014) 1174-1180.

8. H.W. Cheng, X.G. Lu, D.H Hu, Y.W. Zhang, W.Z. Ding, H.L. Zhao, Hydrogen production by catalytic partial oxidation of coke oven gas in $\mathrm{BaCo}_{0.7} \mathrm{Fe}_{0.2} \mathrm{Nb}_{0.1} \mathrm{O}_{3-\delta}$ membranes with surface modification, Int. J. Hydrogen Energy. 36 (2011) 528-538.

9. Z.P. Shao, G.X. Xiong, Y. Cong, W.S. Yang, Synthesis and oxygen permeation study of novel perovskite-type $\mathrm{BaBi}_{x} \mathrm{Co}_{0.2} \mathrm{Fe}_{0.8-x} \mathrm{O}_{3-\delta}$ ceramic membranes, J. Membr. Sci.. 164 (2000) 167-176.

10. H.W. Cheng, W.L. Yao, X.G. Lu, Z.F. Zhou, C.H. Li, J.Z. Liu, Structural stability and oxygen permeability of $\mathrm{BaCo}_{0.7} \mathrm{Fe}_{0.2} \mathrm{M}_{0.1} \mathrm{O}_{3-\delta}(\mathrm{M}=\mathrm{Ta}, \mathrm{Nb}, \mathrm{Zr})$ ceramic membranes for producing hydrogen from coke oven gas, Fuel Process. Technol.. 131 (2015) 36-44.

11. H.W. Cheng, J.Z. Liu, X.G. Lu, W.Z. Ding, Enhancing the Oxygen Permeability of $\mathrm{BaCo}_{0.7} \mathrm{Fe}_{0.2} \mathrm{Nb}_{0.1} \mathrm{O}_{3-\delta}$ Membranes by Coating $\mathrm{GdBaCo}_{2-x} \mathrm{Fe}_{x} \mathrm{O}_{5+\delta}$ for Partial Oxidation of Coke Oven Gas to Syngas, ACS Applied Materials \& Interfaces. 3 (2011) 4032-4039.

12. Y.W. Zhang, K. Su, F.L. Zeng, W.Z. Ding, X.G. Lu, A novel tubular oxygen-permeable membrane reactor for partial oxidation of $\mathrm{CH}_{4}$ in coke oven gas to syngas, Int. J. Hydrogen Energy. 38 (2013) 8783-8789. 